\title{
Correction to: A validated high performance thin layer chromatography method for the simultaneous estimation of berberine, berbamine, palmatine, magnoflorine and jatrorrhizine from Berberis aristata
}

\author{
Ishita A. Basera ${ }^{1,2}$ (D ) Aboli Girme ${ }^{3} \cdot$ Vijay P. Bhatt $^{4} \cdot$ Mamta B. Shah $^{1}$ (I)
}

Published online: 7 September 2021

(c) Akadémiai Kiadó, Budapest, Hungary 2021

\section{Correction to: \\ JPC - Journal of Planar Chromatography - Modern TLC https://doi.org/10.1007/s00764-021-00094-9}

Affiliation has been updated. The original article has been corrected.

\section{Mamta B. Shah}

mbshah2007@ rediffmail.com

1 Department of Pharmacognosy, L. M. College of Pharmacy,

Navrangpura, Ahmedabad, Gujarat 380009, India

2 Gujarat Technological University, Ahmedabad, India

3 Pharmanza Herbal Pvt. Ltd., Anand, Gujarat 388120, India

4 Herbal Research and Development Institute,

Mandal, Gopeshwar, Chamoli, Uttarakhand, India 